Appl. Set-Valued Anal. Optim. 2 (2020), No. 1, pp. 109-121

Available online at http://asvao.biemdas.com

https://doi.org/10.23952/asvao.2.2020.1.07

\title{
ON A CLASS OF NONSMOOTH FRACTIONAL ROBUST MULTI-OBJECTIVE OPTIMIZATION PROBLEMS. PART I: OPTIMALITY CONDITIONS
}

\author{
ZHE HONG ${ }^{1}$, LIGUO JIAO ${ }^{2}$, DO SANG KIM ${ }^{1, *}$ \\ ${ }^{1}$ Department of Applied Mathematics, Pukyong National University, Busan 48513, Korea \\ ${ }^{2}$ School of Mathematical Sciences, Soochow University, Suzhou 215006, China
}

\begin{abstract}
By employing some advanced tools of variational analysis and generalized differentiation, we study the Fritz-John type and Karush-Kuhn-Tucker type necessary optimality conditions for a local (weakly) Pareto solution to a class of nonsmooth fractional robust multi-objective optimization problems. Moreover, sufficient optimality conditions for such a (weakly) Pareto solution to the considered problem are also investigated in light of (strict) generalized convex-concave functions defined in terms of the limiting subdifferential of locally Lipschitz functions.
\end{abstract}

Keywords. Fractional optimization; Robust optimization; Multi-objective optimization; Optimality conditions; Local (weakly) Pareto solution.

\section{INTRODUCTION}

The data of real-world optimization problems is often uncertain, i.e., they are not known exactly when the problem is to be solved $[2,3,4,5]$, due to prediction errors or lack of information. Nowadays, robust optimization has emerged as a remarkable deterministic framework for studying mathematical optimization problems with uncertain data; see $[2,3,4,6,7,8,9$, $13,14,15,16,17,18]$ for theoretical and applied aspects of robust optimization. Among them, Chuong [7] studied optimality conditions (and duality) for robust multi-objective optimization problem involving nonsmooth real-valued functions.

In this paper, we aim to investigate optimality conditions for a class of nonsmooth fractional robust multi-objective optimization problems, which can be regarded as its fractional model of the robust multi-objective optimization problem studied by Chuong [7].

Let $K=\{1, \ldots, l\}, J=\{1, \ldots, m\}$ be index sets, and the functions $p_{k}, q_{k}, k \in K$, be locally Lipschitz on $\mathbb{R}^{n}$, and $g_{j}, j \in J$ be given functions. Furthermore, denote $f(x):=\left(\frac{p_{1}(x)}{q_{1}(x)}, \ldots, \frac{p_{l}(x)}{q_{l}(x)}\right)$ for simplicity. The fractional multi-objective optimization problem with inequality constraints is

$$
\operatorname{Min}_{\mathbb{R}_{+}^{l}}\left\{f(x) \mid g_{j}(x) \leq 0, j \in J\right\},
$$

\footnotetext{
${ }^{*}$ Corresponding author.

E-mail addresses: hongchul1990@gmail.com (Z. Hong), hanchezi@163.com (L.G. Jiao), dskim@ @knu.ac.kr (D.S. Kim).

Received February 1, 2020; Accepted March 5, 2020.
}

(C)2020 Applied Set-Valued Analysis and Optimization 
where $\operatorname{Min}_{\mathbb{R}_{+}^{l}}$ in the above problem is understood with respect to the ordering cone $\mathbb{R}_{+}^{l}:=$ $\left\{\left(y_{1}, \ldots, y_{l}\right) \mid y_{i} \geq 0, i=1, \ldots, l\right\}$; and problem (P) in the face of data uncertainty in the constraints can be written as the following form:

$$
\operatorname{Min}_{\mathbb{R}_{+}^{l}}\left\{f(x) \mid g_{j}\left(x, v_{j}\right) \leq 0, j \in J\right\},
$$

where $x \in \mathbb{R}^{n}$ is the vector of decision variables, $v_{j} \in \mathscr{V}_{j}, j \in J$ are uncertain parameters, $g_{j}$ : $\mathbb{R}^{n} \times \mathscr{V}_{j} \rightarrow \mathbb{R}, j \in J$ are continuous functions.

Following the robust approach, we associate with (UP) its robust counterpart:

$$
\operatorname{Min}_{\mathbb{R}_{+}^{l}}\left\{f(x) \mid g_{j}\left(x, v_{j}\right) \leq 0, \forall v_{j} \in \mathscr{V}_{j}, j \in J\right\} .
$$

Let $F$ be the feasible set of problem (RP), given by

$$
F:=\left\{x \in \mathbb{R}^{n} \mid g_{j}\left(x, v_{j}\right) \leq 0, \forall v_{j} \in \mathscr{V}_{j}, j \in J\right\}
$$

Definition 1.1. We say that $\bar{x} \in F$ is a local Pareto solution to problem (RP) if and only if there is no $x \in F$ and there is neighborhood $U$ of $\bar{x}$ such that

$$
f_{k}(x) \leq f_{k}(\bar{x}), \quad \forall x \in F \cap U, k \in K,
$$

with at least one strict inequality. If in addition all the inequalities in (1.2) are strict, then one has the definition for local weakly Pareto solution to problem (RP).

In what follows, for the sake of convenience, we further assume that $p_{k}(x) \geq 0, q_{k}(x)>0$, $k \in K$ for all $x \in \mathbb{R}^{n}$. Here after, we use the notation $f:=\left(f_{1}, \ldots, f_{l}\right)$, where $f_{k}:=\frac{p_{k}}{q_{k}}, k \in K$, and $g:=\left(g_{1}, \ldots, g_{m}\right)$. Besides, let us make some assumptions for function $g_{j}, j \in J$, given in (1.1). We refer the reader to [7] for more details.

(A1) For a fixed $\bar{x} \in \mathbb{R}^{n}$, there exists $\delta_{j}^{\bar{x}}>0$ such that the function $v_{j} \in \mathscr{V}_{j} \mapsto g_{j}\left(x, v_{j}\right) \in \mathbb{R}$ is upper semicontinuous for each $x \in \mathbb{B}\left(\bar{x}, \delta_{j}^{\bar{x}}\right)$, and the functions $g_{j}\left(\cdot, v_{j}\right), v_{j} \in \mathscr{V}_{j}$, are Lipschitz of given rank $L_{j}>0$ on $\mathbb{B}\left(\bar{x}, \delta_{j}^{\bar{x}}\right)$, i.e.,

$$
\left|g_{j}\left(x_{1}, v_{j}\right)-g_{j}\left(x_{2}, v_{j}\right)\right| \leq L_{j}\left\|x_{1}-x_{2}\right\|, \forall x_{1}, x_{2} \in \mathbb{B}\left(\bar{x}, \delta_{j}^{\bar{x}}\right), \forall v_{j} \in \mathscr{V}_{j} .
$$

(A2) The multifunction $\left(x, v_{j}\right) \in \mathbb{B}\left(\bar{x}, \delta_{j}^{\bar{x}}\right) \times \mathscr{V}_{j} \rightrightarrows \partial_{x} g_{j}\left(x, v_{j}\right) \subset \mathbb{R}^{n}$ is closed at $\left(\bar{x}, \bar{v}_{j}\right)$ for each $\bar{v}_{j} \in \mathscr{V}_{j}(\bar{x})$, where the symbol $\partial_{x}$ stands for the limiting subdifferential operation with respect to $x$, and the notation $\mathscr{V}_{j}(\bar{x})$ signifies active indices in $\mathscr{V}_{j}$ at $\bar{x}$, i.e.,

$$
\mathscr{V}_{j}(\bar{x}):=\left\{v_{j} \in \mathscr{V}_{j} \mid g_{j}\left(\bar{x}, v_{j}\right)=G_{j}(\bar{x})\right\}
$$

with $G_{j}(\bar{x}):=\sup _{v_{j} \in \mathscr{V}_{j}} g_{j}\left(\bar{x}, v_{j}\right)$.

Now, using the parametric approach, we transform the problem (RP) into the nonsmooth non-fractional robust multi-objective optimization problem $(\mathrm{RP})_{\gamma}$ with a parametric $\gamma \in \mathbb{R}_{+}^{l}$.

$$
\operatorname{Min}_{\mathbb{R}_{+}^{l}}\left\{\hat{f}(x):=\left(p_{1}(x)-\gamma_{1} q_{1}(x), \ldots, p_{l}(x)-\gamma_{l} q_{l}(x)\right) \mid x \in F\right\},
$$

where the feasible set $F$ is same as (1.1).

The following result describes the relation between the (weakly) Pareto solutions to problem (RP) and its associate non-fractional model. 
Lemma 1.1. (c.f. [1, Lemma 1]) Let $\bar{x} \in F$ be a local (weakly) Pareto solution to problem (RP). Then there exists $\bar{\gamma} \in \mathbb{R}_{+}^{l}$, such that $\bar{x} \in F$ is also a local (weakly) Pareto solution to problem $(\mathrm{RP})_{\bar{\gamma}}$. If $\bar{x} \in F$ is a local (weakly) Pareto solution to problem $(\mathrm{RP})_{\bar{\gamma}}$ with $\bar{\gamma}_{k}=\frac{p_{k}(\bar{x})}{q_{k}(\bar{x})}$, then $\bar{x}$ be a local (weakly) Pareto solution to problem (RP).

The rest of the paper is organized as follows. In Section 2, some preliminaries and previous results are provided. Section 3 shows (both necessary and sufficient) optimality conditions for a class of nonsmooth fractional robust multi-objective optimization problems. Finally, conclusions are given in Section 4.

\section{PRELIMINARIES}

In this section, we recall some notations and preliminary results that will be used throughout this paper; see, e.g., $[19,20]$. Let $\mathbb{R}^{n}$ denote the Euclidean space equipped with the usual Euclidean norm $\|\cdot\|$. The nonnegative orthant of $\mathbb{R}^{n}$ is denoted by $\mathbb{R}_{+}^{n}:=\left\{\left(x_{1}, \ldots, x_{n}\right) \mid x_{i} \geq\right.$ $0, i=1, \ldots, n\}$. The inner product in $\mathbb{R}^{n}$ is defined by $\langle x, y\rangle:=x^{T} y$ for all $x, y \in \mathbb{R}^{n}$. The symbol $\mathbb{B}(x, \rho)$ stands for the open ball centered at $x \in \mathbb{R}^{n}$ with the radius $\rho>0$. For a given set $\Omega \subset \mathbb{R}^{n}$, we use conv $\Omega$ to indicate the convex hull of $\Omega$, and the notation $x \stackrel{\Omega}{\rightarrow} \bar{x}$ means that $x \rightarrow \bar{x}$ with $x \in \Omega$.

A given set-valued mapping $F: \Omega \subset \mathbb{R}^{n} \rightrightarrows \mathbb{R}^{m}$ is said to be closed at $\bar{x} \in \Omega$ if for any sequence $\left\{x_{k}\right\} \subset \Omega, x_{k} \rightarrow \bar{x}$, and any sequence $\left\{y_{k}\right\} \subset \mathbb{R}^{m}, y_{k} \rightarrow \bar{y}$, one has $\bar{y} \in F(\bar{x})$.

Given a multifuction $F: \mathbb{R}^{n} \rightrightarrows \mathbb{R}^{m}$ with values $F(x) \subset \mathbb{R}^{m}$ in the collection of all the subsets of $\mathbb{R}^{m}$. The limiting construction

$$
\operatorname{Limsup}_{x \rightarrow \bar{x}} F(x):=\left\{y \in \mathbb{R}^{m} \mid \exists x_{k} \rightarrow \bar{x}, y_{k} \rightarrow y \text { with } y_{k} \in F\left(x_{k}\right) \text { for all } k \in \mathbb{N}:=\{1,2, \ldots\}\right\}
$$

is known as the Painlevé-Kuratowski upper/outer limit of $F$ at $\bar{x}$.

Given $\Omega \subset \mathbb{R}^{n}$, and $\bar{x} \in \Omega$, define the collection of Fréchet/regular normal cone to $\Omega$ at $\bar{x}$ by

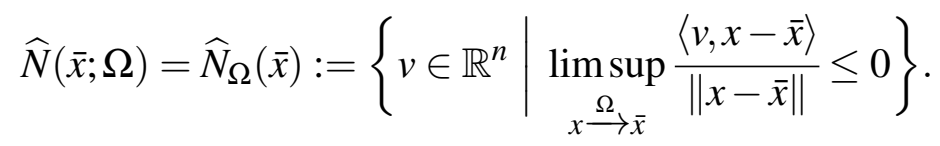

If $\bar{x} \notin \Omega$, we put $\widehat{N}(\bar{x} ; \Omega):=\emptyset$.

The Mordukhovich/limiting normal cone $N(\bar{x} ; \Omega)$ to $\Omega$ at $\bar{x} \in \Omega \subset \mathbb{R}^{n}$ is obtained from regular normal cones by taking the sequential Painlevé-Kurotowski upper limits as

$$
N(\bar{x} ; \Omega):=\underset{x \stackrel{\Omega}{\rightarrow}_{\bar{x}}}{\operatorname{Limsup}} \widehat{N}(x ; \Omega) .
$$

If $\bar{x} \notin \Omega$, we put $N(\bar{x} ; \Omega):=\emptyset$.

For an extended real-valued function $\phi: \mathbb{R}^{n} \rightarrow \overline{\mathbb{R}}:=[-\infty,+\infty]$ its domain and epigraph are defined by

$$
\operatorname{dom} \phi:=\left\{x \in \mathbb{R}^{n} \mid \phi(x)<\infty\right\} \quad \text { and } \quad \text { epi } \phi:=\left\{(x, \mu) \in \mathbb{R}^{n} \times \mathbb{R} \mid \phi(x) \leq \mu\right\} .
$$

Let $\phi: \mathbb{R}^{n} \rightarrow \overline{\mathbb{R}}$ be finite at $\bar{x} \in \operatorname{dom} \phi$, then the collection of basic subgradients, or the (basic/Mordukhovich/limiting) subdifferential, of $\phi$ at $\bar{x}$ is defined by

$$
\partial \phi(\bar{x}):=\left\{v \in \mathbb{R}^{n} \mid(v,-1) \in N((\bar{x}, \phi(\bar{x})) ; \text { epi } \phi)\right\} \text {. }
$$


Given $\Omega \subset \mathbb{R}^{n}$, and consider the indicator function $\delta(\cdot ; \Omega)$ defined by

$$
\delta(x ; \Omega)=\left\{\begin{array}{cl}
0, & \text { if } x \in \Omega \\
+\infty, & \text { otherwise }
\end{array}\right.
$$

Furthermore, we have a relation between the Mordukhovich normal cone and the limiting subdifferential of the indicator function as follows (see [20, Proposition 1.19]):

$$
N(\bar{x} ; \Omega)=\partial \delta(\bar{x} ; \Omega), \quad \forall \bar{x} \in \Omega .
$$

Recall that a function $\phi: \mathbb{R}^{n} \rightarrow \overline{\mathbb{R}}$ is known as locally Lipschitz at $\bar{x} \in \mathbb{R}^{n}$ with rank $L>0$, i.e., there exists $\rho>0$ such that

$$
\left\|\phi\left(x_{1}\right)-\phi\left(x_{2}\right)\right\| \leq L\left\|x_{1}-x_{2}\right\|, \forall x_{1}, x_{2} \in \mathbb{B}(\bar{x}, \rho) .
$$

The generalized Fermat's rule is formulated as follows (see [20, Proposition 1.30]).

Lemma 2.1. Let $\phi: \mathbb{R}^{n} \rightarrow \overline{\mathbb{R}}$ be finite at $\bar{x}$. If $\bar{x}$ is a local minimizer of $\phi$, then

$$
0 \in \partial \phi(\bar{x})
$$

We close this section by recalling the following lemmas, which will be very useful for establishing optimality conditions; see [20].

Lemma 2.2. [20, Corollary 2.21 and Theorem 4.10 (ii)]

(i) (sum rule) Let $\phi_{i}: \mathbb{R}^{n} \rightarrow \overline{\mathbb{R}}, i=1,2, \ldots, m, m \geq 2$ be lower semicontinuous around $\bar{x} \in \mathbb{R}^{n}$, and let all but one of these functions be Lipschitz continuous around $\bar{x}$. Then

$$
\partial\left(\phi_{1}+\phi_{2}+\ldots+\phi_{m}\right)(\bar{x}) \subset \partial \phi_{1}(\bar{x})+\partial \phi_{2}(\bar{x})+\ldots+\partial \phi_{m}(\bar{x}) .
$$

(ii) (max-function rule) Let $\phi_{i}: \mathbb{R}^{n} \rightarrow \overline{\mathbb{R}}, i=1,2, \ldots, m, m \geq 2$ be lower semicontinuous around $\bar{x}$ for $i \in I_{\max }(\bar{x})$ and be upper semicontinuous at $\bar{x}$ for $i \notin I_{\max }(\bar{x})$, suppose that each $\phi_{i}, i=1, \ldots, m$, is Lipschitz continuous around $\bar{x}$. Then we have the inclusion

$$
\partial\left(\max \phi_{i}\right)(\bar{x}) \subset \bigcup\left\{\partial\left(\sum_{i \in I_{\max }(\bar{x})} \lambda_{i} \phi_{i}\right)(\bar{x}) \mid\left(\lambda_{1}, \ldots, \lambda_{m}\right) \in \Lambda(\bar{x})\right\},
$$

where the equality holds and the maximum functions are lower regular at $\bar{x}$ if each $\phi_{i}$ is lower regular at this point and sets $I_{\max }(\bar{x})$ and $\Lambda(\bar{x})$ are defined as follows:

$$
\begin{aligned}
& I_{\max }(\bar{x}):=\left\{i \in\{1, \ldots, m\} \mid \phi_{i}(\bar{x})=\left(\max \phi_{i}\right)(\bar{x})\right\}, \\
& \Lambda(\bar{x}):=\left\{\left(\lambda_{1}, \ldots, \lambda_{m}\right) \mid \lambda_{i} \geq 0, \sum_{i=1}^{m} \lambda_{i}=1, \lambda_{i}\left(\phi_{i}(\bar{x})-\left(\max \phi_{i}\right)(\bar{x})\right)=0\right\} .
\end{aligned}
$$

Lemma 2.3. [20, Corollary 4.8] Let $\phi_{i}: \mathbb{R}^{n} \rightarrow \overline{\mathbb{R}}$ for $i=1,2$ be Lipschitz continuous around $\bar{x}$ with $\phi_{2}(\bar{x}) \neq 0$. Then we have

$$
\partial\left(\frac{\phi_{1}}{\phi_{2}}\right)(\bar{x}) \subset \frac{\partial\left(\phi_{2}(\bar{x}) \phi_{1}\right)(\bar{x})-\partial\left(\phi_{1}(\bar{x}) \phi_{2}\right)(\bar{x})}{\left[\phi_{2}(\bar{x})\right]^{2}} .
$$




\section{Optimality Conditions}

This section is devoted to studying optimality conditions for the nonsmooth fractional robust multi-objective optimization problem (RP). More precisely, by exploiting the nonsmooth version of Fermat's rule, the mean value inequality, and the sum rule for the limiting subdifferential, we first establish necessary conditions for local (weakly) Pareto solution to problem (RP). Then, by introducing concepts of generalized convex-concavity for a family of real-valued functions, we establish sufficient conditions for such solutions.

3.1. Necessary Optimality Conditions. In what follows, we establish the Fritz-John type necessary optimality condition for a local weakly Pareto solution to the associated nonsmooth nonfractional robust multi-objective optimization problem $(\mathrm{RP}) \gamma$.

Theorem 3.1 (Fritz-John type). Let $\gamma_{k}=\frac{p_{k}(\bar{x})}{q_{k}(\bar{x})}, k \in K$. If $\bar{x}$ is a local weakly Pareto solution to problem (RP), then there exist $\beta_{k} \geq 0, k \in K$ and $\mu_{j} \geq 0, j \in J$ with $\sum_{k \in K} \beta_{k}+\sum_{j \in J} \mu_{j}=1$ such that

$$
\left\{\begin{array}{l}
0 \in \sum_{k \in K} \beta_{k} \partial p_{k}(\bar{x})-\sum_{k \in K} \beta_{k} \gamma_{k} \partial q_{k}(\bar{x})+\sum_{j \in J} \mu_{j} \operatorname{conv}\left\{\cup \partial_{x} g_{j}\left(\bar{x}, v_{j}\right) \mid v_{j} \in \mathscr{V}_{j}(\bar{x})\right\} \\
\mu_{j} \sup _{v_{j} \in \mathscr{V}_{j}} g_{j}\left(\bar{x}, v_{j}\right)=0, j \in J .
\end{array}\right.
$$

Proof. Let $\bar{x}$ be a local weakly Pareto solution to problem (RP). For $j \in J$, we consider $\delta_{j}^{\bar{x}}, L_{j}$, $\mathscr{V}_{j}(\bar{x})$, and $G_{j}(\bar{x})$ as in assumption (A1) and (A2). First, along with the proof of [7, Theorem 3.3], we admit that

$$
\operatorname{conv}\left\{\cup \partial_{x} g_{j}\left(\bar{x}, v_{j}\right) \mid v_{j} \in \mathscr{V}_{j}(\bar{x})\right\}
$$

is closed and compact. Moreover, with the help of the mean value inequality (see [20, Corollary 4.14(ii)], it can be shown that

$$
\partial G_{j}(\bar{x}) \subset \operatorname{conv}\left\{\cup \partial_{x} g_{j}\left(\bar{x}, v_{j}\right) \mid v_{j} \in \mathscr{V}_{j}(\bar{x})\right\} .
$$

Since $\bar{x}$ is a local weakly Pareto solution to problem (RP). Then, by Lemma 1.1, $\bar{x}$ is a weakly Pareto solution to problem $(\mathrm{RP}) \gamma$. Now, define a real-valued function $\psi$ on $\mathbb{R}^{n}$ by

$$
\psi(x):=\max _{k \in K, j \in J}\left\{p_{k}(x)-\gamma_{k} q_{k}(x), G_{j}(x)\right\}, \quad x \in \mathbb{R}^{n} .
$$

We claim that

$$
\psi(\bar{x})=0 \leq \psi(x), \quad \forall x \in U .
$$

Indeed, it is easy to see the equality in (3.3) holds due to $\bar{x} \in F$. If $x \in F \cap U$, then $\psi(x) \geq 0$. Otherwise $\psi(x)<0$ leads us to that

$$
p_{k}(x)-\gamma_{k} q_{k}(x)<0, \quad \text { for all } k \in K \text {. }
$$

Equivalently,

$$
\frac{p_{k}(x)}{q_{k}(x)}-\frac{p_{k}(\bar{x})}{q_{k}(\bar{x})}<0, \quad \text { for all } k \in K,
$$

which is a contradiction to the fact that $\bar{x}$ is a local weakly Pareto solution to problem (RP). If $x \in U \backslash F$, then there exists $j_{0} \in\{1, \ldots, m\}$ such that $G_{j_{0}}(x)>0$, which entails that $\psi(x)>0$. 
We assert by (3.3) that $\bar{x}$ is a minimizer of the following scalar problem

$$
\min _{x \in U} \psi(x) .
$$

Invoking now the nonsmooth version of Fermat's rule (2.1) to problem (3.4), we have

$$
0 \in \partial \psi(\bar{x})
$$

Applying further the formula for the limiting subdifferential of maximum functions and the limiting subdifferential sum rule for Lipschitz functions (see Lemma 2.2), one has

$$
\begin{aligned}
\partial \psi(\bar{x}) & =\partial\left(\max _{k \in K, j \in J}\left\{p_{k}(\cdot)-\gamma_{k} q_{k}(\cdot), G_{j}(\cdot)\right\}\right)(\bar{x}) \\
& \subset \bigcup\left\{\partial\left(\sum_{k \in K} \beta_{k}\left(p_{k}(\cdot)-\gamma_{k} q_{k}(\cdot)\right)+\sum_{j \in J} \mu_{j} G_{j}(\cdot)\right)(\bar{x}) \mid\left(\beta_{1}, \ldots, \beta_{l}, \mu_{1}, \ldots, \mu_{m}\right) \in \Lambda(\bar{x})\right\} \\
& \subset \bigcup\left\{\sum_{k \in K} \beta_{k} \partial p_{k}(\bar{x})-\sum_{k \in K} \beta_{k} \gamma_{k} \partial q_{k}(\bar{x})+\sum_{j \in J} \mu_{j} \partial G_{j}(\bar{x}) \mid\left(\beta_{1}, \ldots, \beta_{l}, \mu_{1}, \ldots, \mu_{m}\right) \in \Lambda(\bar{x})\right\},
\end{aligned}
$$

and we get

$$
\begin{aligned}
0 \in\left\{\sum_{k \in K} \beta_{k} \partial p_{k}(\bar{x})-\right. & \sum_{k \in K} \beta_{k} \gamma_{k} \partial q_{k}(\bar{x})+\sum_{j \in J} \mu_{j} \partial G_{j}(\bar{x}) \\
& \left.\mid\left(\beta_{1}, \ldots, \beta_{l}, \mu_{1}, \ldots, \mu_{m}\right) \in \Lambda(\bar{x}), \mu_{j} G_{j}(\bar{x})=0\right\} .
\end{aligned}
$$

From (3.2) together with (1.3), we derive

$$
\begin{array}{r}
0 \in\left\{\sum_{k \in K} \beta_{k} \partial p_{k}(\bar{x})-\sum_{k \in K} \beta_{k} \gamma_{k} \partial q_{k}(\bar{x})+\sum_{j \in J} \mu_{j} \operatorname{conv}\left\{\cup \partial_{x} g_{j}\left(\bar{x}, v_{j}\right) \mid v_{j} \in \mathscr{V}_{j}(\bar{x})\right\}\right. \\
\left.\mid\left(\beta_{1}, \ldots, \beta_{l}, \mu_{1}, \ldots, \mu_{m}\right) \in \Lambda(\bar{x}), \mu_{j} \sup _{v_{j} \in \mathscr{V}_{j}} g_{j}\left(\bar{x}, v_{j}\right)=0\right\},
\end{array}
$$

which establishes (3.1), and completes the proof.

To obtain the necessary optimality condition of Karush-Kuhn-Tucker type for a local weakly Pareto solution to problem (RP), the following constraint qualification is needed, see Chuong [7].

Definition 3.1. Let $\bar{x} \in F$. We say that the constraint qualification (CQ) is satisfied at $\bar{x}$ if

$$
0 \notin \operatorname{conv}\left\{\cup \partial_{x} g_{j}\left(\bar{x}, v_{j}\right) \mid v_{j} \in \mathscr{V}_{j}(\bar{x}), j \in J\right\} .
$$

Remark 3.1. If the (CQ) condition holds, then $\beta_{k}$ in (3.1) can be chosen not all zero, and hence, a point $\bar{x} \in F$ is satisfy (KKT) condition (3.1). Indeed, if $\beta_{k}=0$, for all $k \in K$, then $0 \in \operatorname{conv}\left\{\cup \partial_{x} g_{j}\left(\bar{x}, v_{j}\right) \mid v_{j} \in \mathscr{V}_{j}(\bar{x}), j \in J\right\}$, which reaches to a contradiction to our assumption that the $(\mathrm{CQ})$ condition holds.

Now, under the satisfaction of the (CQ) condition, we provide the Kurash-Kuhn-Tucker type necessary optimality condition for a local weakly Pareto solution to the nonsmooth fractional robust multi-objective optimization problem (RP). 
Theorem 3.2 (KKT type). Let $\bar{x}$ be a local weakly Pareto solution to problem (RP), then there exist $\beta_{k} \geq 0, k \in K$ and $\mu_{j} \geq 0, j \in J$ with $\sum_{k \in K} \beta_{k}+\sum_{j \in J} \mu_{j}=1$ such that

$$
\left\{\begin{array}{l}
0 \in \sum_{k \in K} \alpha_{k}\left(\partial p_{k}(\bar{x})-\frac{p_{k}(\bar{x})}{q_{k}(\bar{x})} \partial q_{k}(\bar{x})\right)+\sum_{j \in J} \mu_{j} \operatorname{conv}\left\{\cup \partial_{x} g_{j}\left(\bar{x}, v_{j}\right) \mid v_{j} \in \mathscr{V}_{j}(\bar{x})\right\} \\
\mu_{j} \sup _{v_{j} \in \mathscr{V}_{j}} g_{j}\left(\bar{x}, v_{j}\right)=0, j \in J
\end{array}\right.
$$

where $\alpha_{k}=\frac{\beta_{k}}{q_{k}(\bar{x})}$. If we further assume that the (CQ) condition holds at $\bar{x}$, then $\sum_{k \in K} \alpha_{k} \neq 0$.

Proof. Let $\bar{x}$ be a local weakly Pareto solution to problem (RP). For $j \in J$, we consider $\delta_{j}^{\bar{x}}, L_{j}$, $\mathscr{V}_{j}(\bar{x})$, and $G_{j}(\bar{x})$ as in assumptions (A1) and (A2). Again, with the proof of [7, Theorem 3.3], we admit that

$$
\operatorname{conv}\left\{\cup \partial_{x} g_{j}\left(\bar{x}, v_{j}\right) \mid v_{j} \in \mathscr{V}_{j}(\bar{x})\right\}
$$

is closed and compact. Moreover, with the help of the mean value inequality (see [20, Corollary 4.14(ii)]), it can be shown that

$$
\partial G_{j}(\bar{x}) \subset \operatorname{conv}\left\{\cup \partial_{x} g_{j}\left(\bar{x}, v_{j}\right) \mid v_{j} \in \mathscr{V}_{j}(\bar{x})\right\}
$$

Since $\bar{x}$ is a local weakly Pareto solution to problem (RP) and define a real-valued function $\psi$ on $\mathbb{R}^{n}$ by

$$
\psi(x):=\max _{k \in K, j \in J}\left\{\frac{p_{k}(x)}{q_{k}(x)}-\frac{p_{k}(\bar{x})}{q_{k}(\bar{x})}, G_{j}(x)\right\}, \quad x \in \mathbb{R}^{n} .
$$

We are going to show that

$$
\psi(\bar{x})=0 \leq \psi(x), \quad \forall x \in U
$$

Indeed, it is easy to see the equality in (3.7) holds due to $\bar{x} \in F$. If $x \in F \cap U$, then $\psi(x) \geq 0$. Otherwise $\psi(x)<0$ leads us to that

$$
\frac{p_{k}(x)}{q_{k}(x)}-\frac{p_{k}(\bar{x})}{q_{k}(\bar{x})}<0, \quad \text { for all } k \in K,
$$

which is a contradiction to the fact that $\bar{x}$ is a local weakly Pareto solution problem (RP). If $x \in U \backslash F$, then there exists $j_{0} \in\{1, \ldots, m\}$ such that $G_{j_{0}}(x)>0$, which entails that $\psi(x)>0$.

We assert by (3.7) that $\bar{x}$ is a minimizer of the following scalar problem

$$
\min _{x \in U} \psi(x)
$$

Invoking now the nonsmooth version of Fermat's rule (2.1) to problem (3.8), we have

$$
0 \in \partial \psi(\bar{x})
$$


Applying further the formula for the limiting subdifferential of maximum functions and the limiting subdifferential sum rule for Lipschitz functions (see Lemma 2.2), it yields that

$$
\begin{aligned}
\partial \psi(\bar{x}) & =\partial\left(\max _{k \in K, j \in J}\left\{\frac{p_{k}}{q_{k}}(\cdot)-\frac{p_{k}(\bar{x})}{q_{k}(\bar{x})}, G_{j}(\cdot)\right\}\right)(\bar{x}) \\
& \subset \bigcup\left\{\partial\left(\sum_{k \in K} \beta_{k}\left\{\frac{p_{k}}{q_{k}}(\cdot)-\frac{p_{k}(\bar{x})}{q_{k}(\bar{x})}\right\}+\sum_{j \in J} \mu_{j} G_{j}(\cdot)\right)(\bar{x}) \mid\left(\beta_{1}, \ldots, \beta_{l}, \mu_{1}, \ldots, \mu_{m}\right) \in \Lambda(\bar{x})\right\} \\
& \subset \bigcup\left\{\sum_{k \in K} \beta_{k} \partial\left(\frac{p_{k}}{q_{k}}\right)(\bar{x})+\sum_{j \in J} \mu_{j} \partial G_{j}(\bar{x}) \mid\left(\beta_{1}, \ldots, \beta_{l}, \mu_{1}, \ldots, \mu_{m}\right) \in \Lambda(\bar{x})\right\},
\end{aligned}
$$

and we get

$$
0 \in\left\{\sum_{k \in K} \beta_{k} \partial\left(\frac{p_{k}}{q_{k}}\right)(\bar{x})+\sum_{j=1}^{J} \mu_{j} \partial G_{j}(\bar{x}) \mid\left(\beta_{1}, \ldots, \beta_{l}, \mu_{1}, \ldots, \mu_{m}\right) \in \Lambda(\bar{x}), \mu_{j} G_{j}(\bar{x})=0\right\} .
$$

It follows from (3.6) and (1.3) that

$$
\begin{aligned}
0 \in\left\{\sum_{k \in K} \beta_{k} \partial\left(\frac{p_{k}}{q_{k}}\right)(\bar{x})+\sum_{j \in J} \mu_{j} \operatorname{conv}\left\{\cup \partial_{x} g_{j}\left(\bar{x}, v_{j}\right) \mid v_{j} \in \mathscr{V}_{j}(\bar{x})\right\}\right. \\
\left.\mid\left(\beta_{1}, \ldots, \beta_{l}, \mu_{1}, \ldots, \mu_{m}\right) \in \Lambda(\bar{x}), \mu_{j} \sup _{v_{j} \in \mathscr{V}_{j}} g_{j}\left(\bar{x}, v_{j}\right)=0\right\} .
\end{aligned}
$$

Taking (2.2) into account, we arrive at

$$
\begin{aligned}
\sum_{k \in K} \beta_{k} \partial\left(\frac{p_{k}}{q_{k}}\right)(\bar{x}) & \subset \sum_{k \in K} \beta_{k} \frac{\partial\left(q_{k}(\bar{x}) p_{k}\right)(\bar{x})-\partial\left(p_{k}(\bar{x}) q_{k}\right)(\bar{x})}{\left[q_{k}(\bar{x})\right]^{2}} \\
& =\sum_{k \in K} \beta_{k} \frac{q_{k}(\bar{x}) \partial p_{k}(\bar{x})-p_{k}(\bar{x}) \partial q_{k}(\bar{x})}{\left[q_{k}(\bar{x})\right]^{2}} \\
& =\sum_{k \in K} \frac{\beta_{k}}{q_{k}(\bar{x})}\left(\partial p_{k}(\bar{x})-\frac{p_{k}(\bar{x})}{q_{k}(\bar{x})} \partial q_{k}(\bar{x})\right) .
\end{aligned}
$$

By letting $\alpha_{k}:=\frac{\beta_{k}}{q_{k}(\bar{x})}$ for $k \in K$, we observe that (3.5) is obtained by (3.9) and (3.10). Moreover, it is clear that $\sum_{k \in K} \alpha_{k} \neq 0$ providing the $(\mathrm{CQ})$ condition is satisfied at $\bar{x}$. Thus, the proof follows.

3.2. Sufficient Optimality Conditions. In order to formulate sufficient conditions for a (weakly) Pareto solution to problem (RP), we give concepts of (strictly) generalized convex-concavity type at a given point for locally Lipschitz functions. It is worth mentioning that the definition below is motivated by [7, Definition 3.9], [12, Definition 3.7] and [11, Definition 3.11].

Definition 3.2. (i) We say that $(p, g ; q)$ is generalized convex-concave at $\bar{x} \in \mathbb{R}^{n}$ if for any $x \in \mathbb{R}^{n}, \xi_{k} \in \partial p_{k}(\bar{x}), \zeta_{k} \in \partial q_{k}(\bar{x}), k \in K$, and $\eta_{v} \in \partial_{x} g_{j}(\bar{x}, v), v \in \mathscr{V}_{j}(\bar{x}), j \in J$, there exists $h \in \mathbb{R}^{n}$ such that

$$
\begin{array}{rlrl}
p_{k}(x)-p_{k}(\bar{x}) & \geq\left\langle\xi_{k}, h\right\rangle, & & k \in K, \\
q_{k}(x)-q_{k}(\bar{x}) & \leq\left\langle\zeta_{k}, h\right\rangle, \quad & k \in K, \\
g_{j}(x, v)-g_{j}(\bar{x}, v) & \geq\left\langle\eta_{v}, h\right\rangle, \quad & v \in \mathscr{V}_{j}(\bar{x}), j \in J,
\end{array}
$$


where $\mathscr{V}_{j}(\bar{x}), j \in J$, are defined as in (1.3).

(ii) We say that $(p, g ; q)$ is strictly generalized convex-concave at $\bar{x} \in \mathbb{R}^{n}$ if for any $x \in$ $\mathbb{R}^{n} \backslash\{\bar{x}\}, \xi_{k} \in \partial p_{k}(\bar{x}), \zeta_{k} \in \partial q_{k}(\bar{x}), k \in K$, and $\eta_{v} \in \partial_{x} g_{j}(\bar{x}, v), v \in \mathscr{V}_{j}(\bar{x}), j \in J$, there exists $h \in \mathbb{R}^{n}$ such that

$$
\begin{aligned}
p_{k}(x)-p_{k}(\bar{x}) & >\left\langle\xi_{k}, h\right\rangle, & & k \in K, \\
q_{k}(x)-q_{k}(\bar{x}) & \leq\left\langle\zeta_{k}, h\right\rangle, & & k \in K, \\
g_{j}(x, v)-g_{j}(\bar{x}, v) & \geq\left\langle\eta_{v}, h\right\rangle, & & v \in \mathscr{V}_{j}(\bar{x}), j \in J,
\end{aligned}
$$

where $\mathscr{V}_{j}(\bar{x}), j \in J$, are defined as in (1.3).

Remark 3.2. We see that if $p_{k}, k \in K$, and $g_{j}, j \in J$ are convex (resp., strictly convex), and $q_{k}$, $k \in K$ are concave (strictly concave), then $(p, g ; q)$ is generalized convex-concave (resp., strictly generalized convex-concave) on $\mathbb{R}^{n}$ at any $\bar{x} \in \mathbb{R}^{n}$ with $h:=x-\bar{x}$ for each $x \in \mathbb{R}^{n}$.

Theorem 3.3. Assume that $\bar{x} \in F$ satisfies the condition (3.1) with $\sum_{k \in K} \beta_{k} \neq 0$.

(i) If $(p, g ; q)$ is generalized convex-concave at $\bar{x}$, then $\bar{x}$ is a weakly Pareto solution to problem (RP).

(ii) If $(p, g ; q)$ is strictly generalized convex-concave at $\bar{x}$, then $\bar{x}$ is a Pareto solution to problem (RP).

Proof. Since $\bar{x} \in F$ satisfies the condition (3.1), there exist $\beta_{k} \geq 0, \xi_{k} \in \partial p_{k}(\bar{x}), \zeta_{k} \in \partial q_{k}(\bar{x})$, $\gamma_{k}=\frac{p_{k}(\bar{x})}{q_{k}(\bar{x})}, k \in K$ with $\sum_{k \in K} \beta_{k} \neq 0$, and $\mu_{j} \geq 0, j \in J, \mu_{j i} \geq 0, \eta_{j i} \in \partial_{x} g_{j}\left(\bar{x}, v_{j i}\right), v_{j i} \in \mathscr{V}_{j}(\bar{x})$, $i \in I_{j}=\left\{1, \ldots, i_{j}\right\}, i_{j} \in \mathbb{N}, \sum_{i \in I_{j}} \mu_{j i}=1$, such that

$$
\begin{array}{r}
\sum_{k \in K} \beta_{k} \xi_{k}-\sum_{k \in K} \beta_{k} \gamma_{k} \zeta_{k}+\sum_{j \in J} \mu_{j}\left(\sum_{i \in I_{j}} \mu_{j i} \eta_{j i}\right)=0, \\
\mu_{j} \sup _{v_{j} \in \mathscr{Y}_{j}} g_{j}\left(\bar{x}, v_{j}\right)=0, j \in J .
\end{array}
$$

We first justify (i). Assume to the contrary that $\bar{x}$ is not a weakly Pareto solution to problem (RP), then there is $\hat{x} \in F$ such that

$$
\frac{p_{k}(\hat{x})}{q_{k}(\hat{x})}-\frac{p_{k}(\bar{x})}{q_{k}(\bar{x})}<0, \quad \forall k \in K .
$$

Equivalently,

$$
p_{k}(\hat{x})-\gamma_{k} q_{k}(\hat{x})<0, \quad \forall k \in K
$$

where $\gamma_{k}=\frac{p_{k}(\bar{x})}{q_{k}(\bar{x})}$, and note the hidden fact that $q_{k}(\hat{x})>0, k \in K$.

By the generalized convex-concavity of $(p, g ; q)$ at $\bar{x}$, we deduce from (3.11) that for such $\hat{x}$ there is $h \in \mathbb{R}^{n}$ such that

$$
\begin{aligned}
0 & =\sum_{k \in K} \beta_{k}\left\langle\xi_{k}, h\right\rangle-\sum_{k \in K} \beta_{k} \gamma_{k}\left\langle\zeta_{k}, h\right\rangle+\sum_{j \in J} \mu_{j}\left(\sum_{i \in I_{j}} \mu_{j i}\left\langle\eta_{j i}, h\right\rangle\right) \\
& \leq \sum_{k \in K} \beta_{k}\left(p_{k}(\hat{x})-p_{k}(\bar{x})\right)-\sum_{k \in K} \beta_{k} \gamma_{k}\left(q_{k}(\hat{x})-q_{k}(\bar{x})\right)+\sum_{j \in J} \mu_{j}\left(\sum_{i \in I_{j}} \mu_{j i}\left[g_{j}\left(\hat{x}, v_{j i}\right)-g_{j}\left(\bar{x}, v_{j i}\right)\right]\right) \\
& =\sum_{k \in K} \beta_{k} p_{k}(\hat{x})-\sum_{k \in K} \beta_{k} \gamma_{k} q_{k}(\hat{x})+\sum_{j \in J} \mu_{j}\left(\sum_{i \in I_{j}} \mu_{j i}\left[g_{j}\left(\hat{x}, v_{j i}\right)-g_{j}\left(\bar{x}, v_{j i}\right)\right]\right) .
\end{aligned}
$$


Hence,

$$
0 \leq \sum_{k \in K} \beta_{k}\left[p_{k}(\hat{x})-\gamma_{k} q_{k}(\hat{x})\right]+\sum_{j \in J} \mu_{j}\left(\sum_{i \in I_{j}} \mu_{j i}\left[g_{j}\left(\hat{x}, v_{j i}\right)-g_{j}\left(\bar{x}, v_{j i}\right)\right]\right)
$$

In addition, $v_{j i} \in \mathscr{V}_{j}(\bar{x})$,

$$
g_{j}\left(\bar{x}, v_{j i}\right)=\sup _{v_{j} \in \mathscr{V}_{j}} g_{j}\left(\bar{x}, v_{j}\right), \quad \forall j \in J, \forall i \in I_{j} .
$$

Thus, it follows from (3.12) that $\mu_{j} g_{j}\left(\bar{x}, v_{j i}\right)=0$ for $j \in J$ and $i \in I_{j}$. In addition, due to $\hat{x} \in F$, $\mu_{j} g_{j}\left(\hat{x}, v_{j i}\right) \leq 0$ for $j \in J$ and $i \in I_{j}$. So, we get by (3.14) that

$$
0 \leq \sum_{k \in K} \beta_{k}\left[p_{k}(\hat{x})-\gamma_{k} q_{k}(\hat{x})\right]
$$

This entails that there is $k_{0} \in K$ such that

$$
0 \leq p_{k_{0}}(\hat{x})-\gamma_{k_{0}} q_{k_{0}}(\hat{x})
$$

due to $\beta_{k} \geq 0, k \in K$ with $\sum_{k \in K} \beta_{k} \neq 0$. This contradicts (3.13) and the proof of (i) follows.

Now, we prove (ii). Suppose to the contrary that $\bar{x}$ is not a Pareto solution to problem (RP), then there is $\hat{x} \in F$ such that

$$
\frac{p_{k}(\hat{x})}{q_{k}(\hat{x})}-\frac{p_{k}(\bar{x})}{q_{k}(\bar{x})} \leq 0, \quad \forall k \in K,
$$

with at least one strict inequality. Equivalently,

$$
p_{k}(\hat{x})-\gamma_{k} q_{k}(\hat{x}) \leq 0, \quad \forall k \in K,
$$

with at least one strict inequality, where $\gamma_{k}=\frac{p_{k}(\bar{x})}{q_{k}(\bar{x})}$, and fact that $q_{k}(\hat{x})>0, k \in K$.

By the strictly generalized convex-concavity of $(p, g ; q)$ at $\bar{x}$, we deduce from (3.11) that for such $\hat{x}$ there is $h \in \mathbb{R}^{n}$ such that

$$
\begin{aligned}
0 & =\sum_{k \in K} \beta_{k}\left\langle\xi_{k}, h\right\rangle-\sum_{k \in K} \beta_{k} \gamma_{k}\left\langle\zeta_{k}, h\right\rangle+\sum_{j \in J} \mu_{j}\left(\sum_{i \in I_{j}} \mu_{j i}\left\langle\eta_{j i}, h\right\rangle\right) \\
& <\sum_{k \in K} \beta_{k}\left(p_{k}(\hat{x})-p_{k}(\bar{x})\right)-\sum_{k \in K} \beta_{k} \gamma_{k}\left(q_{k}(\hat{x})-q_{k}(\bar{x})\right)+\sum_{j \in J} \mu_{j}\left(\sum_{i \in I_{j}} \mu_{j i}\left[g_{j}\left(\hat{x}, v_{j i}\right)-g_{j}\left(\bar{x}, v_{j i}\right)\right]\right) \\
& =\sum_{k \in K} \beta_{k} p_{k}(\hat{x})-\sum_{k \in K} \beta_{k} \gamma_{k} q_{k}(\hat{x})+\sum_{j \in J} \mu_{j}\left(\sum_{i \in I_{j}} \mu_{j i}\left[g_{j}\left(\hat{x}, v_{j i}\right)-g_{j}\left(\bar{x}, v_{j i}\right)\right]\right) .
\end{aligned}
$$

This (thanks to $\beta_{k} \geq 0, k \in K$ with $\sum_{k \in K} \beta_{k} \neq 0$ ) entails that there is $k_{0} \in K$ such that

$$
0<p_{k_{0}}(\hat{x})-\gamma_{k_{0}} q_{k_{0}}(\hat{x})
$$

which contradicts (3.15). Therefore, the proof of (ii) is complete.

Theorem 3.4. Assume that $\bar{x} \in F$ satisfies the condition (3.5) with $\sum_{k \in K} \alpha_{k} \neq 0$.

(iii) If $(p, g ; q)$ is generalized convex-concave at $\bar{x}$, then $\bar{x}$ is a weakly Pareto solution to problem (RP).

(iv) If $(p, g ; q)$ is strictly generalized convex-concave at $\bar{x}$, then $\bar{x}$ is a Pareto solution to problem (RP). 
Proof. Since $\bar{x} \in F$ satisfies the condition (3.5), there exist $\alpha_{k} \geq 0, \xi_{k} \in \partial p_{k}(\bar{x}), \zeta_{k} \in \partial q_{k}(\bar{x})$, $k \in K$ with $\sum_{k \in K} \alpha_{k} \neq 0$, and $\mu_{j} \geq 0, j \in J, \mu_{j i} \geq 0, \eta_{j i} \in \partial_{x} g_{j}\left(\bar{x}, v_{j i}\right), v_{j i} \in \mathscr{V}_{j}(\bar{x}), i \in I_{j}=$ $\left\{1, \ldots, i_{j}\right\}, i_{j} \in \mathbb{N}, \sum_{i \in I_{j}} \mu_{j i}=1$, such that

$$
\begin{array}{r}
\sum_{k \in K} \alpha_{k}\left(\xi_{k}-\frac{p_{k}(\bar{x})}{q_{k}(\bar{x})} \zeta_{k}\right)+\sum_{j \in J} \mu_{j}\left(\sum_{i \in I_{j}} \mu_{j i} \eta_{j i}\right)=0, \\
\mu_{j} \sup _{v_{j} \in \mathscr{V}_{j}} g_{j}\left(\bar{x}, v_{j}\right)=0, j \in J .
\end{array}
$$

We first justify (iii). Assume to the contrary that $\bar{x}$ is not a weakly Pareto solution to problem (RP), then there is $\hat{x} \in F$ such that

$$
\frac{p_{k}(\hat{x})}{q_{k}(\hat{x})}-\frac{p_{k}(\bar{x})}{q_{k}(\bar{x})}<0, \quad \forall k \in K .
$$

By the generalized convex-concavity of $(p, g ; q)$ at $\bar{x}$, we deduce from (3.16) that for such $\hat{x}$ there is $h \in \mathbb{R}^{n}$ such that

$$
\begin{aligned}
0 & =\sum_{k \in K} \alpha_{k}\left(\left\langle\xi_{k}, h\right\rangle-\frac{p_{k}(\bar{x})}{q_{k}(\bar{x})}\left\langle\zeta_{k}, h\right\rangle\right)+\sum_{j \in J} \mu_{j}\left(\sum_{i \in I_{j}} \mu_{j i}\left\langle\eta_{j i}, h\right\rangle\right) \\
& \leq \sum_{k \in K} \alpha_{k}\left[p_{k}(\hat{x})-p_{k}(\bar{x})-\frac{p_{k}(\bar{x})}{q_{k}(\bar{x})}\left(q_{k}(\hat{x})-q_{k}(\bar{x})\right)\right]+\sum_{j \in J} \mu_{j}\left(\sum_{i \in I_{j}} \mu_{j i}\left[g_{j}\left(\hat{x}, v_{j i}\right)-g_{j}\left(\bar{x}, v_{j i}\right)\right]\right) \\
& =\sum_{k \in K} \alpha_{k}\left[p_{k}(\hat{x})-\frac{p_{k}(\bar{x})}{q_{k}(\bar{x})} q_{k}(\hat{x})\right]+\sum_{j \in J} \mu_{j}\left(\sum_{i \in I_{j}} \mu_{j i}\left[g_{j}\left(\hat{x}, v_{j i}\right)-g_{j}\left(\bar{x}, v_{j i}\right)\right]\right) .
\end{aligned}
$$

Hence,

$$
0 \leq \sum_{k \in K} \alpha_{k}\left[p_{k}(\hat{x})-\frac{p_{k}(\bar{x})}{q_{k}(\bar{x})} q_{k}(\hat{x})\right]+\sum_{j \in J} \mu_{j}\left(\sum_{i \in I_{j}} \mu_{j i}\left[g_{j}\left(\hat{x}, v_{j i}\right)-g_{j}\left(\bar{x}, v_{j i}\right)\right]\right) .
$$

In addition, $v_{j i} \in \mathscr{V}_{j}(\bar{x})$,

$$
g_{j}\left(\bar{x}, v_{j i}\right)=\sup _{v_{j} \in \mathscr{V}_{j}} g_{j}\left(\bar{x}, v_{j}\right), \quad \forall j \in J, \forall i \in I_{j} .
$$

Thus, it follows from (3.17) that $\mu_{j} g_{j}\left(\bar{x}, v_{j i}\right)=0$ for $j \in J$ and $i \in I_{j}$. In addition, due to $\hat{x} \in F$, $\mu_{j} g_{j}\left(\hat{x}, v_{j i}\right) \leq 0$ for $j \in J$ and $i \in I_{j}$. So, we get by (3.19) that

$$
0 \leq \sum_{k \in K} \alpha_{k}\left[p_{k}(\hat{x})-\frac{p_{k}(\bar{x})}{q_{k}(\bar{x})} q_{k}(\hat{x})\right] \text {. }
$$

This entails that there is $k_{0} \in K$ such that

$$
0 \leq p_{k_{0}}(\hat{x})-\frac{p_{k_{0}}(\bar{x})}{q_{k_{0}}(\bar{x})} q_{k_{0}}(\hat{x})
$$

due to $\alpha_{k} \geq 0, k \in K$ with $\sum_{k \in K} \alpha_{k} \neq 0$. The inequality in (3.20) is equivalent to the following one

$$
\frac{p_{k_{0}}(\bar{x})}{q_{k_{0}}(\bar{x})} \leq \frac{p_{k_{0}}(\hat{x})}{q_{k_{0}}(\hat{x})}
$$

which contradicts (3.18). Thus, the proof of (iii) is complete. 
Now, we show (iv). Suppose to the contrary that $\bar{x}$ is not a Pareto solution to problem (RP), then there is $\hat{x} \in F$ such that

$$
\frac{p_{k}(\hat{x})}{q_{k}(\hat{x})}-\frac{p_{k}(\bar{x})}{q_{k}(\bar{x})} \leq 0, \quad \forall k \in K,
$$

with at least one strict inequality.

By the strictly generalized convex-concavity of $(p, g ; q)$ at $\bar{x}$, we deduce from (3.16) that for such $\hat{x}$ there is $h \in \mathbb{R}^{n}$ such that

$$
\begin{aligned}
0 & =\sum_{k \in K} \alpha_{k}\left(\left\langle\xi_{k}, h\right\rangle-\frac{p_{k}(\bar{x})}{q_{k}(\bar{x})}\left\langle\zeta_{k}, h\right\rangle\right)+\sum_{j \in J} \mu_{j}\left(\sum_{i \in I_{j}} \mu_{j i}\left\langle\eta_{j i}, h\right\rangle\right) \\
& <\sum_{k \in K} \alpha_{k}\left[p_{k}(\hat{x})-p_{k}(\bar{x})-\frac{p_{k}(\bar{x})}{q_{k}(\bar{x})}\left(q_{k}(\hat{x})-q_{k}(\bar{x})\right)\right]+\sum_{j \in J} \mu_{j}\left(\sum_{i \in I_{j}} \mu_{j i}\left[g_{j}\left(\hat{x}, v_{j i}\right)-g_{j}\left(\bar{x}, v_{j i}\right)\right]\right) \\
& =\sum_{k \in K} \alpha_{k}\left[p_{k}(\hat{x})-\frac{p_{k}(\bar{x})}{q_{k}(\bar{x})} q_{k}(\hat{x})\right]+\sum_{j \in J} \mu_{j}\left(\sum_{i \in I_{j}} \mu_{j i}\left[g_{j}\left(\hat{x}, v_{j i}\right)-g_{j}\left(\bar{x}, v_{j i}\right)\right]\right) .
\end{aligned}
$$

This entails that there is $k_{0} \in K$ such that

$$
0<p_{k_{0}}(\hat{x})-\frac{p_{k_{0}}(\bar{x})}{q_{k_{0}}(\bar{x})} q_{k_{0}}(\hat{x})
$$

due to $\alpha_{k} \geq 0, k \in K$ with $\sum_{k \in K} \alpha_{k} \neq 0$. Equivalently,

$$
\frac{p_{k_{0}}(\bar{x})}{q_{k_{0}}(\bar{x})}<\frac{p_{k_{0}}(\hat{x})}{q_{k_{0}}(\hat{x})},
$$

which contradicts (3.21) and the proof of (iv) follows.

\section{CONCLUSiOns}

In 2014, Chuong and Kim [10] studied optimality conditions and duality for nonsmooth multi-objective optimization problems by employing some advanced tools of variational analysis and generalized differentiation. Then, the optimality conditions and duality for nonsmooth robust multi-objective optimization problems were obtained in 2016 by Chuong [7]. In this paper, we further investigated optimality conditions for a class of nonsmooth fractional robust multi-objective optimization problems.

The result on duality for the class of nonsmooth fractional robust multi-objective optimization problems modeled by (RP) will be examined in a forthcoming study.

\section{Acknowledgments}

The authors would like to express their sincere thanks to anonymous referees for valuable suggestions and comments for the paper.

\section{REFERENCES}

[1] C.R. Bector, S. Chandra, C. Singh, Duality in Multiobjective Fractional Programming. In: A. Cambini, E. Castagnoli, L. Martein, P. Mazzoleni, S. Schaible (eds.) Generalized Convexity and Fractional Programming with Economic Applications, pp. 232-241. Springer, Berlin, 1990.

[2] A. Beck, A. Ben-Tal, Duality in robust optimization: primal worst equals dual best, Oper. Res. Lett. 37 (2009), 1-6. 
[3] A. Ben-Tal, A. Nemirovski, Robust convex optimization, Math. Oper. Res. 23 (1998), 769-805.

[4] A. Ben-Tal, A. Nemirovski, Selected topics in robust convex optimization, Math. Program. 112 (2008), 125158.

[5] A. Ben-Tal, L. El Ghaoui, A. Nemirovski, Robust Optimization, Princeton University Press, NJ, 2009.

[6] A. Ben-Tal, D. den Hertog, J. Vial, Deriving robust counterparts of nonlinear uncertain inequalities, Math. Program. 149 (2015), 265-299.

[7] T.D. Chuong, Optimality and duality for robust multiobjective optimization problems, Nonlinear Anal. 134 (2016), 127-143.

[8] T.D. Chuong, Robust alternative theorem for linear inequalities with applications to robust multiobjective optimization, Oper. Res. Lett. 45 (2017), 575-580.

[9] T.D. Chuong, Linear matrix inequality conditions and duality for a class of robust multiobjective convex polynomial programs, SIAM J. Optim. 28 (2018), 2466-2488.

[10] T.D. Chuong, D.S. Kim, Optimality conditions and duality in nonsmooth multiobjective optimization problems, Ann. Oper. Res. 217 (2014), 117-136.

[11] T.D. Chuong, D.S. Kim, Approximate solutions of multiobjective optimization problems, Positivity 20 (2016), 187-207.

[12] T.D. Chuong, D.S. Kim, A class of nonsmooth fractional multiobjective optimization problems, Ann. Oper. Res. 244 (2016), 367-383.

[13] L. El Ghaoui, H. Lebret, Robust solution to least-squares problems with uncertain data, SIAM J. Matrix Anal. Appl. 18 (1997), 1035-1064.

[14] L. El Ghaoui, F. Oustry, H. Lebret, Robust solutions to uncertain semidefinite programs, SIAM J. Optim. 9 (1998), 33-52.

[15] L.G. Jiao, J.H. Lee, Approximate optimality and approximate duality for quasi approximate solutions in robust convex semidefinite programs, J. Optim. Theory Appl. 176 (2018), 74-93.

[16] L.G. Jiao, J.H. Lee, Finding efficient solutions in robust multiple objective optimization with SOS-convex polynomial data, Ann. Oper. Res. (2019) doi: 10.1007/s10479-019-03216-z

[17] G.M. Lee, P.T. Son, On nonsmooth optimality theorems for robust optimization problems, Bull. Korean Math. Soc. 51 (2014), 287-301.

[18] J.H. Lee, L.G. Jiao, On quasi $\varepsilon$-solution for robust convex optimization problems, Optim. Lett. 11 (2017), 1609-1622.

[19] B.S. Mordukhovich, Variational Analysis and Generalized Differentiation, I: Basic Theory, Springer Verlag, 2006.

[20] B.S. Mordukhovich, Variational Analysis and Applications, Springer Monographs in Mathematics, 2018. 